Psychological Medicine, 2007, 37, 1820. (C) 2007 Cambridge University Press doi:10.1017/S003329170700219X Printed in the United Kingdom

\title{
NOTICEBOARD
}

\section{New look for Psychological Medicine}

The December issue marks the last appearance of the Journal in its current format, which dates back to the 1970s. The Journal's appearance has been updated and from January 2008 it will appear in a larger format with a new cover. 